Ciência e Natura, Santa Maria, v. 37 Ed. Especial PROFMAT, 2015, p.179-191

Revista do Centro de Ciências Naturais e Exatas - UFSM

ISSN impressa: 0100-8307 ISSN on-line: 2179-460X

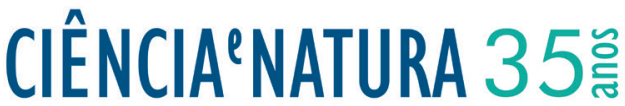

\section{As Cônicas na Geometria do Taxi}

\author{
The Conics in Taxicab Geometry
}

\author{
Carlos Augusto Gomes Loiola*1, Christine Sertã Costa ${ }^{2}$
}

${ }^{1}$ Me. Profmat Puc-Rio, SEEDUC-RJ, Brasil

${ }^{2}$ Dra. Engenharia de Produção, Matemática, PUC-Rio, Colégio Pedro II, Brasil

\begin{abstract}
Resumo
O presente trabalho tem como objetivo principal apresentar as cônicas quando definidas sob uma geometria não euclidiana: a Geometria do Táxi. A escolha desta geometria se deu em função da simplicidade das suas definições possibilitando aplicações diversas na Educação Básica. Ela difere da geometria euclidiana por sua métrica e apresenta resultados interessantes e surpreendentes que possibilitam o desenvolvimento de uma aprendizagem mais crítica e significativa.
\end{abstract}

Palavras-chave: Euclides; Quinto Postulado; Geometria do Taxi; Lugar Geométrico; Ensino

\begin{abstract}
This paper aims to present conics when defined in a non-Euclidean geometry: the Taxicab geometry. The choice of this geometry was due to the simplicity of its definitions enabling diverse applications in Basic Education. It differs from Euclidean geometry by its metric and presents interesting and surprising results that enable the development of a more critical and meaningful learning.
\end{abstract}

Keywords: Euclid; Fifth Postulate; Non-Euclidean Geometry; Taxicab Geometry; Locus; Education. 


\section{Introdução}

Há cerca de 2400 anos, Aristóteles deve ter passado muito tempo observando os navios que se distanciavam do cais e desapareciam no horizonte. E num daqueles momentos que se perdem na história deve ter se perguntado o motivo pelo qual os navios iam sumindo primeiramente os cascos e depois as velas. Em uma Terra plana os navios deveriam diminuir por igual. Só havia uma explicação para tal evidência: a Terra é redonda. Em relação a esse fato Mlodinow nos afirma que para observar a estrutura de nosso planeta em grande escala, Aristóteles tinha olhado através da janela da Geometria.

Ao estudar geometria, ou melhor, ao olhar pela janela da geometria, como diria Mlodinow, nossos alunos tem a oportunidade de apreciar e interagir com o ambiente ao seu redor podendo relacionar as ideias geométricas com números, medições e estruturas matemáticas, e desse modo ampliar o seu horizonte de conhecimento. A geometria pode assim agir como tema integrador na Matemática do Ensino Médio uma vez que a intuição e o formalismo, a abstração e a dedução fazem parte de sua estrutura. Além disso, os Parâmetros Curriculares Nacionais estabelecem que a Matemática no Ensino Médio deve não só ter um aspecto formativo e instrumental, mas também ser apresentada como uma ciência.

A Matemática no Ensino Médio tem um valor formativo, que ajuda a estruturar o pensamento e o raciocínio dedutivo, porém também desempenha um papel instrumental, pois é uma ferramenta que serve para a vida cotidiana e para muitas tarefas específicas em quase todas as atividades humanas.

Contudo a Matemática no Ensino Médio deve ser vista como uma ciência, com suas características estruturais específicas. É importante que o aluno perceba que as definições, demonstrações e encadeamentos conceituais e lógicos tem a função de construir novos conceitos e estruturas a partir de outros e que servem para validar intuições e dar sentido às técnicas aplicadas. (PCN Parte - III, 1998, pg.40)
Em continuidade os Parâmetros Curriculares Nacionais afirmam que:

Cabe à Matemática do Ensino Médio apresentar ao aluno conhecimento de novas informações e instrumentos necessários para que seja possível a ele continuar aprendendo. Saber aprender é condição básica para prosseguir aperfeiçoando-se ao longo da vida. (PCN Parte - III, 1998, pg.41)

Pautados nesta referência é que organizamos o presente trabalho que, de modo simplista, apresenta as características estruturais e definições de uma teoria, até então desconhecida por grande parte do alunado da educação básica e, a partir delas, utiliza encadeamentos lógicos para a obtenção de novas estruturas. Pretendemos com esse trabalho não só valorizar perante os alunos a importância das definições em uma estrutura matemática, mas também apresentar a eles um novo olhar a partir da utilização dessas definições, permitindo que também utilizem esse modo de pensar em outras situações.

Uma contextualização histórica acerca da Geometria Euclidiana e do surgimento de geometrias não Euclidianas é apresentada e, posteriormente, as definições e conceituações básicas da Geometria do Taxi são descritas. O mote principal do presente estudo está no paralelo estabelecido entre as equações e representações gráficas da circunferência, elipse, parábola e hipérbole nas geometrias Euclidiana e do Taxi.

\section{Um Pouco de História}

No istmo, entre o mar Mediterrâneo e o Lago Mareótis, a oeste do delta do rio Nilo, em uma antiga aldeia de pescadores e pastores chamada Rhakotis, nasceu a cidade de Alexandria. Em suas geométricas ruas cuja planificação é atribuída ao urbanista Dinócrates ${ }^{1}$, um homem caminhou por volta de 300 a.E.C. e escreveu um livro que rivaliza com a Bíblia em número de

1 Arquiteto e urbanista originário de Rodes ou Macedônia, viveu na época de Alexandre o Grande, e também construiu a grande pira funerária em Hefestion. 
edições. Esse homem, cuja vida pouco se conhece, chamava-se Euclides e o seu livro, os Elementos.

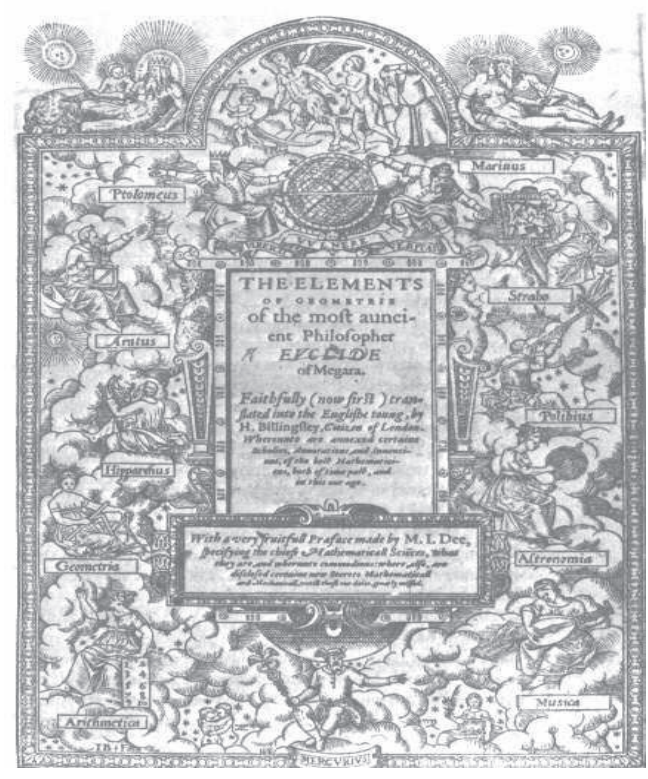

Figura 1 - Capa da primeira edição inglesa de Os Elementos (1570)2

A obra "Os Elementos" é composta de um conjunto de 13 livros. Não são conhecidos registros ou fragmentos do texto original escrito por Euclides, apenas versões e traduções bem tardias foram encontrados, segundo Roque. Porém, não há dúvida quanto à importância do conteúdo apresentado nesses livros e, para muitos autores e pesquisadores o grande mérito está na forma como esse conteúdo é apresentado. Para Tomei Euclides buscou dar a matemática grega uma base sólida utilizando o método axiomático que pela primeira vez pode ser visto atuando em toda a extenção de uma obra.

O método axiomático consiste em um grupo de objetos ou termos não definidos, chamados de objetos ou termos primitivos, em função dos quais todos os demais termos ou objetos são definidos; um conjunto de proposições que se faz sobre os objetos ou termos primitivos e aceitas sem demonstração, que são chamadas de axiomas ou postulados, e finalmente um conjunto de proposições demonstradas utilizando a lógica dedutiva que apresenta as

\footnotetext{
${ }^{2}$ Disponível em:

$<$ http://www.educ.fc.ul.pt/icm/icm2001/icm23/images /elements.jpg $>$. Acesso em 22 de novembro de 2013.
}

propriedades dos objetos ou termos não definidos, que são denominados de teoremas.

Euclides inicia o Livro I dos Elementos citando 23 definições em que procura deixar a compreensão dos objetos e termos, que terão suas propriedades estudadas e estabelecidas no decorrer de sua obra, de forma bem clara e precisa. Algumas definições elencadas por Euclides estão descritas a seguir com tradução de Irineu Bicudo:

1. Ponto é aquilo de que nada é parte.

2. E linha é comprimento sem largura.

3. E extremidade de uma linha são pontos.

4. E uma linha reta é a que está posta por igual com os pontos sobre si mesma.

10. E quando uma reta, tendo sido alteada sobre uma reta, faça os ângulos adjacentes iguais, cada um dos ângulos é reto, e a reta que se alteou é chamada uma perpendicular àquela sobre a qual se alteou.

15. Círculo é uma figura plana contida por uma linha, em relação à qual todas as retas que a encontram, a partir de um ponto dos postos no interior da figura, são iguais entre si.

16. E o ponto é chamado centro do círculo. (Bicudo, 2009, pg.97/98)

Em seguida, Euclides escreve os postulados e axiomas delimitando assim as hipóteses que são utilizadas nas demonstrações dos teoremas e no desenvolvimento de toda a teoria. Os postulados e axiomas estabelecidos por Euclides, também por tradução de Irineu Bicudo, estão descritos a seguir:

Postulados:

1. Fique postulado traçar uma $\operatorname{reta}^{3}$ a partir de todo ponto até todo ponto.

2. Também prolongar uma reta limitada, continuamente, sobre uma reta.

3. E, com todo centro e distância, descrever um círculo.

3 Euclides não faz distinção entre reta e segmento de reta. 
4. E serem iguais entre si todos os ângulos retos.

5. E caso uma reta, caindo sobre duas retas, faça os ângulos interiores e do mesmo lado menores do que dois retos, sendo prolongadas, as duas retas, encontram-se no mesmo lado no qual estão os menores do que dois retos.

Axiomas ou Noções Comuns:

1. As coisas iguais às mesmas coisas são também iguais entre si.

2. E, caso sejam adcionados coisas iguais a coisas iguais, os todos são iguais.

3. E, caso de iguais sejam subtraídas iguais, os restantes são iguais.

4. E, caso iguais sejam adcionados a desiguais, os todos são desiguais.

5. E os dobros da mesma coisa são iguais entre si.

6. E as metades da mesma coisa são iguais entre si.

7. E as coisas que se ajustam uma à outra são iguais entre si.

8. E o todo é maior do que a parte.

9. E duas retas não contêm uma área. (Bicudo, 2009, pg.98/99)

É evidente que, a luz da nossa moderna visão do sistema axiomático, encontramos falhas e enganos cometidos por Euclides na elaboração de suas definições, postulados e axiomas. Entretanto, o questionamento mais importante da obra de Euclides e que permitiu que a matemática expandisse e abrisse novos caminhos e áreas de estudo, girou em torno do Quinto Postulado, também conhecido como Postulado das Paralelas, uma vez que pode ser assim enunciado: Por um ponto, exterior a uma reta, podese traçar uma única reta paralela à reta dada.

Durante muitos anos, nos afirma Aaboe, pesquisadores acreditaram que o Quinto Postulado dependia dos anteriores e, portanto, era possível prová-lo e o mesmo não deveria estar explicitado como um postulado. Várias tentativas de demonstração apareceram e foram, cada uma delas, refutadas.

A obra de Giovanni Girolamo Saccheri (16671733), padre jesuíta, professor de teologia, filosofia e matemática, publicada em 1773 e intitulada "Euclides ab Omni Naevo
Vindicatus" ${ }^{4}$ é mais uma que tem como foco a demonstração do Quinto Postulado de Euclides. A abordagem de Saccheri foi negar o Postulado das Paralelas, ou seja, ele o tomou como falso e aceitou como premissas verdadeiras as 27 primeiras proposições do Livro I de Euclides. A partir daí começou a procurar alguma evidência de que o Quinto Postalado era de fato verdadeiro. Ele utilizou um quadrilátero com dois lados opostos congruentes e perpendiculares à base que hoje conhecemos como quadrilátero de Saccheri estudando assim as suas propriedades. Pode-se dizer que ele descobriu uma nova geometria ou a primeira Geometria Não Euclidiana, sem ter se apercebido de tal fato. Infelizmente Saccheri analisou seus feitos sob uma ótica mais voltada na crença existente na época de que a verdadeira e única geometria era a Euclidiana, do que naquilo que a lógica estava lhe mostrando, deixando assim de caminhar na direção de uma nova e interessante área da matemática. Hoje é considerado como um precursor de uma geometria não euclidiana.

Finalmente, em 1820 um preocupado pai com o futuro profissional do filho lhe dá um conselho por carta: "Não desperdices uma hora no problema. Em vez de ser recompensador, envenenará toda a tua vida". Esse zeloso pai ainda continua tentando dissuadir o filho para outros interesses escrevendo: "os maiores geômetras ponderaram o problema durante centenas de anos e não conseguiram provar o postulado das paralelas sem um novo axioma." E colocando a autoridade de pai: "Acredito que eu mesmo investiguei todas as ideias possíveis...".

Apesar dos apelos de Farkas Wolfgang Bolyai, o jovem de 21 anos, Janos Bolyai, nascido em Kolozsvár, Hungria (atualmente Cluj, Romenia), escreveu ao seu pai em 3 de novembro de 1823 relatando: "Efetuei descobertas maravilhosas que me deixaram extasiados e seria motivo de lamento se as perdesse. Quando as vires, querido pai, também perceberás.". Na mesma carta Janos Bolyai escreve uma conhecida frase "Criei um universo do nada". E como que se desculpando pelo fato de não ter seguido o conselho do pai afirma: "tenho certeza que me trará honra, tal como se já tivesse completado a descoberta".

\footnotetext{
${ }^{4}$ Euclides Livre de Todos os Erros
} 
Uma vez que novas e boas ideias não são privilégio de uma única pessoa, podendo ocorrer de modo simultâneo entre personagens que nunca se conheceram, Farkas Bolyai aconselhou seu filho a agir com rapidez: "primeiro porque as ideias passam facilmente de uns para os outros, que as podem de imediato publicar, e, segundo, há alguma verdade no fato de muitas coisas terem uma época para serem descobertas ao mesmo tempo em vários sítios".

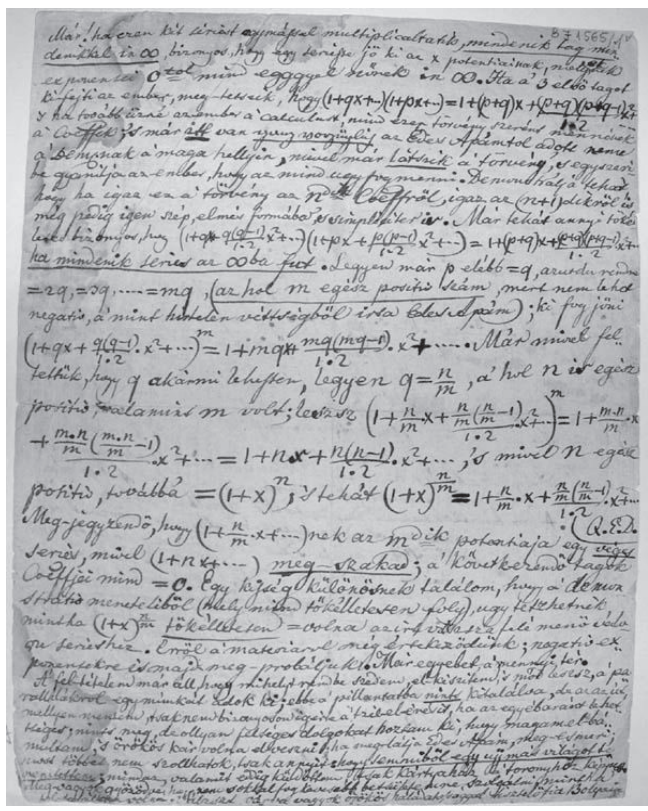

Figura 2- Carta de Janos Bolyai a seu pai ${ }^{5}$

Nessa época Farkas Bolyai trabalhava em seu livro Ensaios sobre os Elementos de Matemática para Jovens Estudiosos e imediatamente convidou Janos Bolyai para incluir nele sua pesquisa. Porém somente em 1832 Janos Bolyai publicou os seus estudos em um apêndice do $1^{\circ}$ volume dos Ensaios de Farkas Bolyai, com o pomposo título "A ciência do espaço absoluto com uma demonstração da independência da verdade ou falsidade do axioma XI de Euclides (que não pode ser decidido a priori) e também a quadratura do círculo no caso de sua falsidade".

Um pouco antes, outro personagem dessa história, Nikolay Ivanovich Lobachevsky, foi o primeiro a publicar um trabalho que apresentou uma geometria não euclidiana, que hoje conhecemos como a Geometria Hiperbólica. Em 1829 em uma desconhecida revista científica russa, O Mensageiro de Kazam, Lobachevsky

\footnotetext{
${ }^{5}$ Disponível em:

http://www.termeszetvilaga.hu/kulonsz/k011/eukleide sz.html. (12/12/13).
}

apresentou um artigo intitulado Sobre os Fundamentos da Geometria. Nesse artigo, escrito em russo, ele relatou todo o desenvolvimento do que chamou de Geometria Imaginária.

Tanto Bolyai quanto Lobachevsky não conheciam o trabalho um do outro e como afirma Mlodinow “... infelizmente, ninguém tampouco sabia. Matemáticos essencialmente obscuros, quando falaram ninguém deu ouvidos". Bolyai nunca mais publicou nenhum outro trabalho e Lobachevsky se tornou reitor da Universidade de Kazam. Mlodinow ainda completa afirmando:

... poderiam ambos ter sumido no limbo desconhecido, não fosse o seu contato com Gauss. Ironicamente, foi a morte de Gauss que finalmente levou à revolução não euclidiana.

Gauss foi um cronista meticuloso das coisas à sua volta. Tinha o prazer de colecionar dados bizarros, tais como a duração da vida de seus amigos mortos, ou o número de passos desde o laboratório onde trabalhava até vários lugares que gostava de visitar. Ele também fazia registros de seu trabalho. Após a sua morte, especialistas estudaram com atenção suas anotações e correspondências. Lá, descobriram a sua pesquisa sobre o espaço não euclidiano, bem como os trabalhos de Bolyai e Lobachevsky. (Mlodinow,2008, pg.125).

Somente em 1862, quando Richard Baltzer, na segunda edição do seu livro Element der Mathmatik incluiu os trabalhos de Bolyai e Lobachevsky, tornou-os referências padrão para aqueles que estudam essas novas geometrias.

Assim, de Euclides por volta de 300 a.E.C até Bolyai e Lobachevsky no século XIX, muito tempo se passou para que a matemática consolidasse as ideias e considerações em torno do Quinto Postulado dos Elementos e abrisse caminho para o estudo de novas geometrias que hoje conhecemos como as Geometrias Não Euclidiana. Tais geometrias são assim chamadas, pois contrariam o Quinto Postulado dos Elementos ou algumas de suas consequências.

Hoje em dia chamamos de Geometria Euclidiana, Geometria Hiperbólica e Geometria Elíptica aquela que adota como o Quinto Postulado respectivamente a afirmação de que 
por um ponto exterior a uma reta pode-se traçar uma única reta paralela, infinitas retas paralelas ou nenhuma reta paralela à reta dada.

A seguir apresentamos uma Geometria Não Euclidiana que por sua simplicidade de compreensão e uso pode ser inserida e trabalhada para contextualizar tópicos do Ensino Básico: A Geometria do Taxi.

\section{A Geometria do Taxi}

A Geometria Euclidiana, amplamente estudada na escola básica, e a Geometria do Taxi, aqui apresentada, diferem de modo conceitual apenas pela métrica. A distância euclidiana é a medida do segmento de reta que une dois pontos quaisquer, sendo determinada pelo Teorema de Pitágoras. A distância do taxi, segundo Krause, é obtida pela soma das medidas dos trajetos horizontais ou verticais entre dois pontos quaisquer dados. Entretanto, sob o olhar da Matemática, essa diferença conceitual ocasiona uma grande distinção e, quando observado do ponto de vista educacional, possibilita o confronto de definições e conceitos com representações geométricas distintas. Tal fato leva a uma saudável discussão e reflexão que acreditamos contribuir para um aprendizado mais significativo e natural.

A matemática define distância como uma função $\mathrm{D}: \mathrm{HxH} \rightarrow \mathrm{R}_{+}$, que associa a cada par ordenado $(x, y)$ pertencentes ao conjunto $\mathrm{HxH}$ um número real não negativo $\mathrm{D}(\mathrm{x}, \mathrm{y})$, chamado a distância de $x$ a y, de modo que tal função, que chamamos de métrica, deve satisfazer as seguintes propriedades:

1. $\mathrm{D}(\mathrm{x}, \mathrm{x})=0$

2. Se $x \neq y$, então $D(x, y)>0$

3. $\mathrm{D}(\mathrm{x}, \mathrm{y})=\mathrm{D}(\mathrm{y}, \mathrm{x})$

4. $\mathrm{D}(\mathrm{x}, \mathrm{z}) \leq \mathrm{D}(\mathrm{x}, \mathrm{y})+\mathrm{D}(\mathrm{y}, \mathrm{z})$ - esta propriedade é conhecida como Desigualdade Triangular em qualquer métrica.

$\mathrm{O}$ conjunto $\mathrm{H}$ ao qual associamos uma métrica $\mathrm{D}: \mathrm{HxH} \rightarrow \mathrm{R}_{+}, \mathrm{D}(\mathrm{x}, \mathrm{y})=\mathrm{k}$, é o que chamamos de espaço métrico e representamos pelo par (H, D).

$\mathrm{O}$ espaço euclidiano $\mathrm{R}^{\mathrm{n}}$ cujos elementos são $\mathrm{x}$ $=\left(x_{1}, x_{2}, \ldots, x_{n}\right)$ onde $x_{i}$ é um número real, é um exemplo de espaço métrico com as seguintes métricas.

$$
\begin{aligned}
& D_{E}(x, y)=\left[\sum_{i=1}^{n}\left(x_{i}-y_{i}\right)^{2}\right]^{1 / 2} \\
& D_{T}(x, y)=\sum_{i=1}^{n}\left|x_{i}-y_{i}\right|
\end{aligned}
$$

A métrica $D_{\text {E }}$ é a que chamamos de Métrica Euclidiana e a $\mathrm{D}_{\mathrm{T}}$ denominamos de Métrica do Taxi.

No particular caso do Plano Euclidiano $\left(\mathrm{R}^{2}\right)$ teríamos a Métrica Euclidiana e a Métrica do Taxi descritas do seguinte modo:

Métrica Euclidiana:

$$
D_{E}(A, B)=\left[\sum_{i=1}^{2}\left(x_{i}-y_{i}\right)^{2}\right]^{1 / 2}=\sqrt{\left(x_{1}-y_{1}\right)^{2}+\left(x_{2}-y_{2}\right)^{2}}
$$

onde $A\left(y_{1}, y_{2}\right) \in B\left(x_{1}, x_{2}\right)$ pertencem a $\mathrm{R}^{2}$.

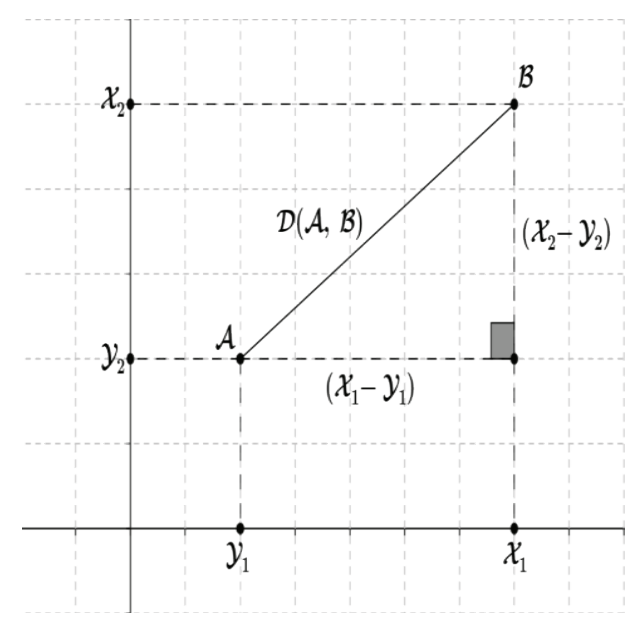

Figura 3 - Métrica Euclidiana

Métrica do Taxi:

$D_{T}(A, B)=\sum_{i=1}^{n}\left|x_{i}-y_{i}\right|=\left|x_{1}-y_{1}\right|+\left|x_{2}-y_{2}\right|$

onde $A\left(y_{1}, y_{2}\right) \in B\left(x_{1}, x_{2}\right)$ pertencem a $\mathrm{R}^{2}$ 


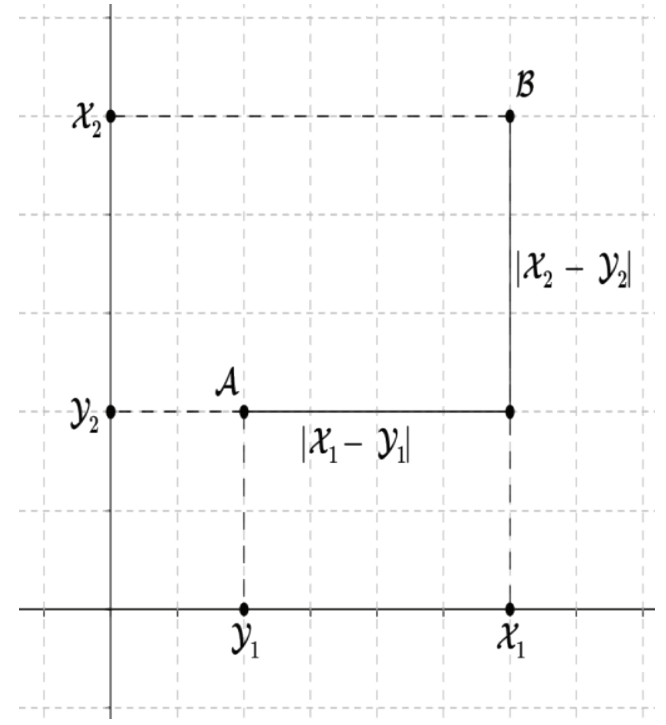

Figura 4 - Métrica do Taxi

A Métrica do Taxi irá induzir uma nova geometria, onde retas e pontos são os mesmos da Geometria Euclidiana e os ângulos são medidos da mesma forma, entretanto os objetos que dependem do conceito de distância se apresentam de forma distinta. Essa nova geometria não euclidiana recebe hoje o nome de Geometria do Taxi, ou Geometria Urbana ou ainda Geometria de Manhattan, e teve como precursor o matemático russo Hermann Minkowski (1884 - 1909) responsável pela definição da métrica do taxi. O termo Geometria do Taxi ou Taxicab Geometry surge pela primeira vez em 1952 quando Karl Menger em uma exibição no Museum of Science and Industry of Chicago apresenta um folheto com o nome You Will Like Geometry em que o termo Taxicab Geometry é usado.

Podemos observar que a Geometria do Taxi é uma Geometria Não Euclidiana no fato que o postulado de congruência do triângulo LAL6 (lado - ângulo- lado) da Geometria Euclidiana, não é válido na Geometria do Taxi. Observe na figura a seguir que os triângulos $\mathrm{ABC}$ e $\mathrm{A}^{\prime} \mathrm{B}^{\prime} \mathrm{C}^{\prime}$ têm respectivamente os lados $A B, A C$ e $A^{\prime} B^{\prime}$, $\mathrm{A}^{\prime} \mathrm{C}^{\prime}$ congruentes, pela métrica do taxi e o ângulo A congruente com o ângulo $\mathrm{A}^{\prime}$, entretanto os triângulos não são congruentes.

6 Se dois triângulos têm ordenadamente congruentes dois lados e o ângulo compreendido então eles são congruentes.

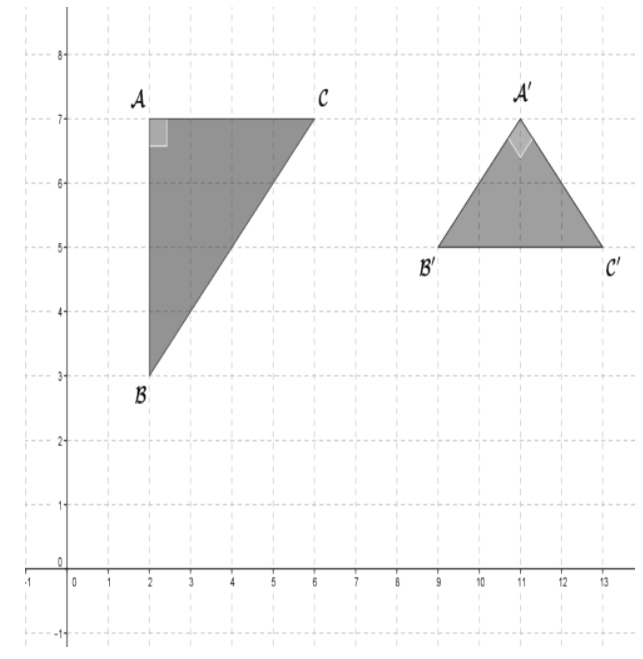

Figura 5 - Negação da congruência LAL

Circunferências, elipses, parábolas e hipérboles são lugares geométricos cujas definições dependem da definição de distância e, portanto se apresentam de forma distinta quando consideramos as geometrias euclidianas e do taxi. Vejamos:

\section{Circunferência}

Conceituamos a circunferência como sendo o lugar geométrico dos pontos do plano que estão a uma mesma distância de um ponto fixo chamado centro. Assim, dados os pontos $\mathrm{M}(\mathrm{x}, \mathrm{y})$ e $\mathrm{O}(\mathrm{a}, \mathrm{b})$, tal que $\mathrm{M}$ pertence a circunferência e $\mathrm{O}$ seja seu centro, $\mathrm{D}(\mathrm{M}, \mathrm{O})=\mathrm{r}$. Observe que:

$$
\begin{aligned}
& C_{E}=\left\{M(x, y) \in R^{2} / D_{E}(O, M)=r\right\} \\
& C_{T}=\left\{M(x, y) \in R^{2} / D_{T}(O, M)=r\right\}
\end{aligned}
$$

E, desse modo:

$$
\begin{aligned}
& C_{E} \equiv \sqrt{(x-a)^{2}+(y-b)^{2}}=r \\
& C_{T} \equiv|x-a|+|y-b|=r
\end{aligned}
$$

Onde $C_{E} \& C_{T}$ representam de modo algébrico a circunferência no modelo euclidiano e no modelo do taxi, respectivamente. A equação $C_{E}$ representada em um sistema de eixos cartesianos nos fornece a seguinte visualização gráfica da circunferência: 


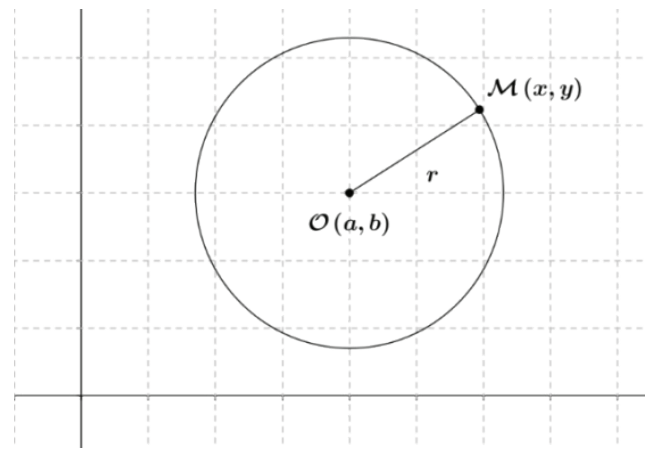

Figura 6 - Circunferência Euclidiana

Analisemos agora a equação:

$$
C_{T} \equiv|x-a|+|y-b|=r:
$$

Para $x<a$ ey $\geq b \rightarrow y=x+(r-a+b)$ (1)

Para $x \geq a$ e $y<b \rightarrow y=-x+(r+a+b)$ (2)

Para $x>a$ e $y \leq b \rightarrow y=x+(-r-a+b)$ (3)

Para $\quad x \leq a \in y<b \rightarrow y=-x+(-r+a+b)$ (4)

Da Geometria Analítica sabemos que as equações (1), (2), (3), e (4) são equações de retas e que os pares de retas (1) e (2), (1) e (4), (3) e (4), (3) e (2) são retas perpendiculares concorrentes nos pontos $\mathrm{A}(\mathrm{a}, \mathrm{b}+\mathrm{r}), \mathrm{B}(\mathrm{a}-\mathrm{r}, \mathrm{b}), \mathrm{C}(\mathrm{a}, \mathrm{b}-\mathrm{r}), \mathrm{D}(\mathrm{a}$ $+r, b)$, respectivamente. Como $\mathrm{D}_{\mathrm{T}}(\mathrm{A}, \mathrm{B})=\mathrm{D}_{\mathrm{T}}(\mathrm{B}$, $C)=\mathrm{D}_{\mathrm{T}}(\mathrm{C}, \mathrm{D})=\mathrm{D} \mathrm{T}(\mathrm{D}, \mathrm{A})=2 \mathrm{r}$, o quadrilátero $A B C D$ é um quadrado. Dessa forma a circunferência na Geometria do Taxi tem como representação gráfica um quadrado cujas diagonais são paralelas aos eixos coordenados, conforme Figura 7.

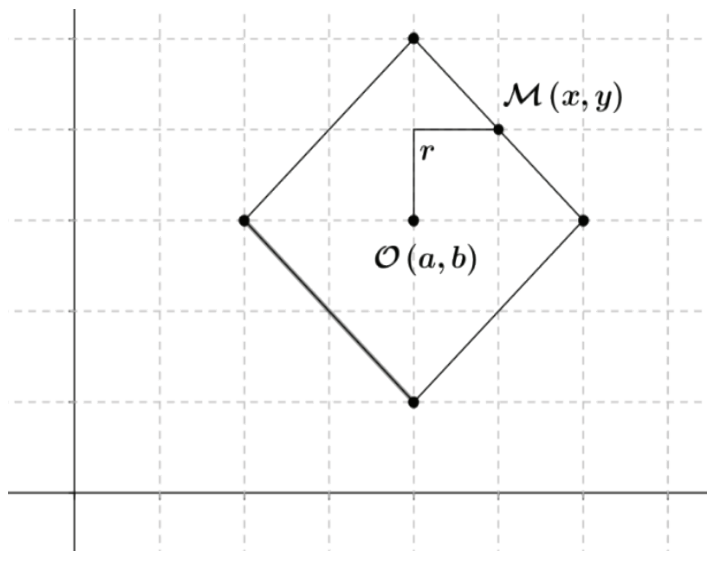

Figura 7 - Circunferência do Taxi

\section{Elipse}

Definimos elipse como sendo o lugar geométrico dos pontos do plano cuja soma das distâncias a dois pontos fixos, chamados de focos, é igual a uma constante, maior do que a distância entre os focos.

Desse modo, dados os pontos $F_{1}(a, b), F_{2}(c, d) \in M(x, y)$ e a constante $k \in R$, temos:

$E_{E}=\left\{M(x, y), \in R^{2} / D_{E}\left(M, F_{1}\right)+D_{E}\left(M, F_{2}\right)=2 k\right\}$

$E_{T}=\left\{M(x, y) \in R^{2} / D_{T}\left(M, F_{1}\right)+D_{T}\left(M, F_{2}\right)=2 k\right\}$

E portanto:

$E_{E} \equiv \sqrt{(x-a)^{2}+(y-b)^{2}}+\sqrt{(x-c)^{2}+(y-d)^{2}}=2 k$

$E_{T} \equiv|x-a|+|y-b|+|x-c|+|y-d|=2 k$

Onde $E_{E} \in E_{T}$ representam de modo algébrico a elipse no modelo euclidiano e no modelo do taxi, respectivamente. A equação $E_{E}$ representada em um sistema de eixos cartesianos nos fornece a seguinte visualização gráfica da elipse:

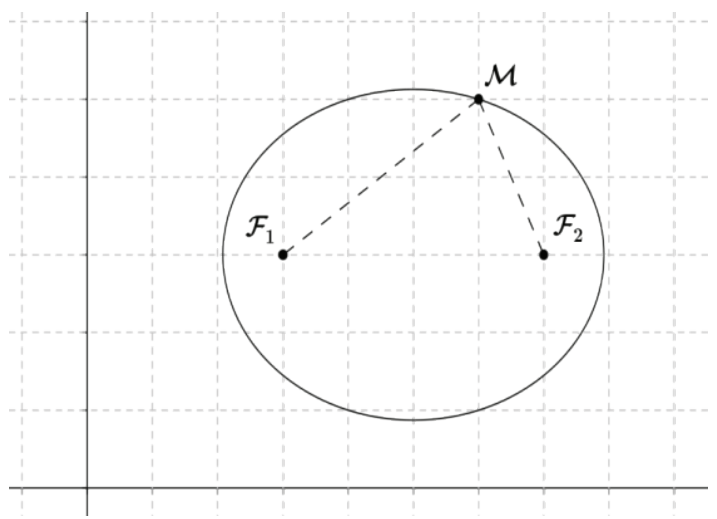

Figura 8 - Elipse Euclidiana

No caso da elipse apresentaremos um exemplo de como obter a sua representação gráfica considerando a geometria do taxi. Cabe ressaltar que embora o método utilizado no exemplo possa ser aplicado em qualquer caso, a representação geométrica obtida pode apresentar outras configurações em função das coordenadas escolhidas para os focos.

Exemplo: Dados os pontos $F_{1}(2,4)$ e $F_{2}(3,6)$, encontre os pontos $M(x, y)$ do plano que atendem a equação:

$$
D_{T}\left(M, F_{1}\right)+D_{T}\left(M, F_{2}\right)=5
$$


Solução:

Nessas condições, temos que:

$|x-2|+|y-4|+|x-3|+|y-6|=5$

Analisando a equação acima, temos os seguintes casos:

Para $x \leq 2$ e $y<4 \rightarrow x+y=5$ (a)

Para $x<2 e 4 \leq y<6 \rightarrow x=1$ (b)

Para $x<2$ e $y \geq 6 \rightarrow-x+y=5$ (c)

Para $2<x \leq 3$ e $y<4 \rightarrow y=3$ (d)

Para

$2<x<3 e 4<y<6 \rightarrow 3=5$ não tem solução

Para $2<x \leq 3$ e $y>6 \rightarrow y=7$ (e)

Para $x>3$ e $y \leq 4 \rightarrow x-y=0$ (f)

Para $x>3 e 4<y \leq 6 \rightarrow x=4(\mathrm{~g})$

Para $x>3$ e $y>6 \rightarrow x+y=10(\mathrm{~h})$

Tomando agora as soluções das equações acima com suas respectivas condições e traçando em um sistema de eixos cartesianos teremos a representação gráfica dessa elipse no modelo do taxi.

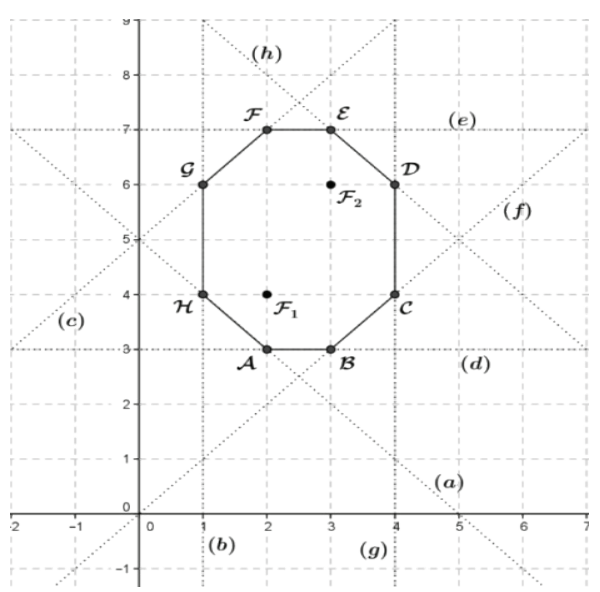

Figura 9 - Elipse do Taxi

\section{Parábola}

Dados uma reta $\mathrm{L}$ e $\mathrm{um}$ ponto $\mathrm{F} \notin \mathrm{L}$, que chamamos de diretriz e foco, respectivamente, definimos uma parábola como sendo o lugar geométrico dos pontos do plano cuja distância ao ponto $\mathrm{F}$ e a L são iguais.

Desse modo dado $\mathrm{L}$ e $\mathrm{F}(\mathrm{a}, \mathrm{b}) \notin \mathrm{L}$, temos que: $P_{E}=\left\{M(x, y) \in R^{2} / D_{E}(M, F)=D_{E}(M, L)\right\}$ $P_{T}=\left\{M(x, y) \in R^{2} / D_{T}(M, F)=D_{T}(M, L)\right\}$

Assim, a definição de parábola depende, além do cálculo da distância entre dois pontos do cálculo da distância entre um ponto e uma reta, que tratamos a seguir.

\section{Distância de Ponto à Reta:}

$\mathrm{Na}$ geometria euclidiana para obter a distância entre um ponto $\mathrm{P}$ e uma reta h pode-se proceder do seguinte modo:

$\mathrm{a}$ - traçar a reta $\mathrm{s}$, perpendicular à reta $\mathrm{h}$, que passe pelo ponto $\mathrm{P}$;

b - marcar o ponto $A$, intersecção da reta $s$ com a reta $\mathrm{h}$;

$\mathrm{c}$ - a distância do ponto $\mathrm{P}$ à reta $\mathrm{h}$ é a medida do segmento PA.

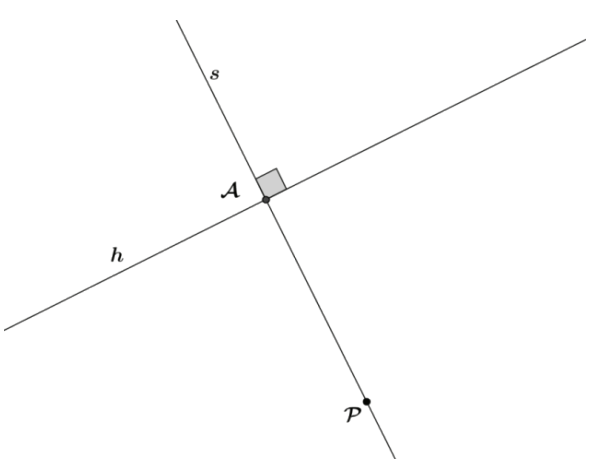

Figura 10 - Distância de Ponto à Reta Geometria Euclidiana - caso 1

Poderíamos também abordar tal situação do seguinte modo:

a - traçar um círculo de centro $\mathrm{P}$ e raio $r<D_{E}(P, h)$;

b - aumentar o círculo até o mesmo intersecctar a reta $\mathrm{h}$ em apenas um ponto, ou seja, até que o círculo seja tangente à reta;

c - a distância do ponto à reta será o raio desse círculo tangente à $h$.

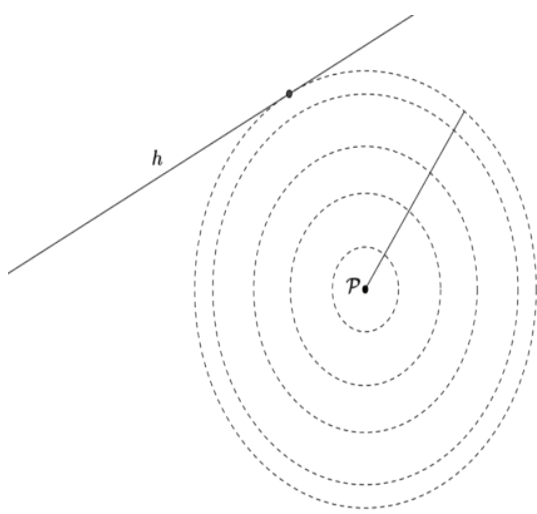

Figura 11 - Distância de Ponto à Reta Geometria Euclidiana - caso 2 
Usaremos o caso 2 para explorar conceitualmente a distância entre uma reta e um ponto na geometria do taxi.

Consideremos então uma reta h e um ponto $\mathrm{P}$ dados, e uma sequência de círculos do taxi concêntricos em $\mathrm{P}$. Três casos podem ocorrer:

Primeiro Caso: A distância do ponto $\mathrm{P}$ à reta h é a distância vertical $D_{T}(P, h)=D_{T}(P, A)$.

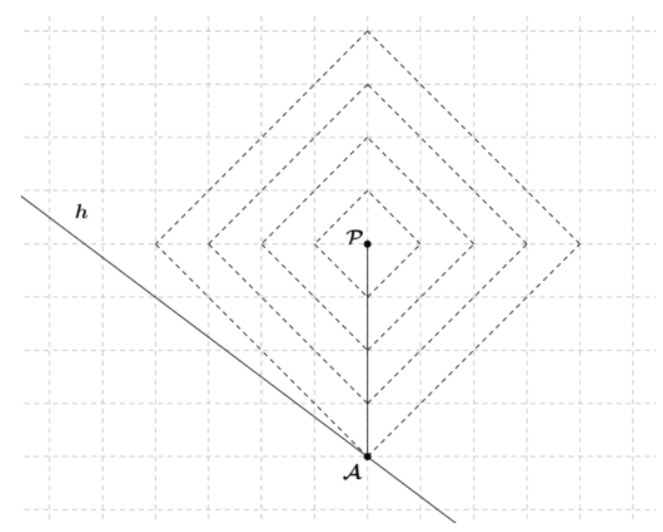

Figura 12 - Distância de Ponto à Reta -

Geometria do Taxi - caso 1

Segundo Caso: A distância do ponto $P$ à reta h é a distância horizontal $D_{T}(P, h)=D_{T}(P, A)$.

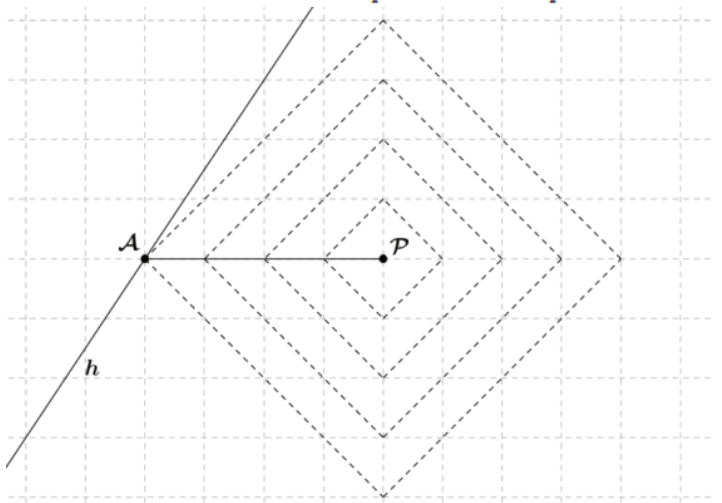

Figura 13 - Distância de Ponto à Reta Geometria do Taxi - caso 2

Terceiro Caso: A distância do ponto $\mathrm{P}$ a reta $\mathrm{h}$ é dada por: $D_{T}(P, h)=D_{T}(P, X), X \in \overline{A_{1} A_{2}}$.

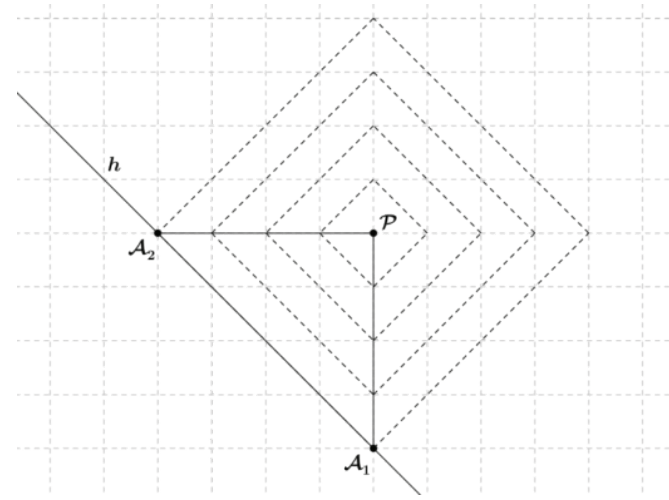

Figura 14 - Distância de Ponto à Reta Geometria do Taxi - caso 3

Com essas observações feitas podemos então demonstrar que: Dado o ponto $P\left(x_{0}, y_{0}\right)$ e a reta $h$ : $a x+b y+c=0$, na geometria do taxi temos que a distância de $\mathrm{P}$ à reta h é dada por:

$$
D_{T}(P, h)=\frac{\left|a x_{0}+b y_{0}+c\right|}{\operatorname{Max} \cdot\{|a|,|b|\}}
$$

Demonstração:

Na geometria do taxi a distância de um ponto a uma reta é definida como: $D_{T}(P, h)=\operatorname{Min}_{X \in h} D_{T}(P, X)$

Sendo $X_{1} \notin X_{2}$ os pontos de intersecção da reta $\mathrm{h}$ com as retas $\quad x=x_{0}$ e $y=y_{0}$, respectivamente, tem-se:

$D_{T}(P, h)=\operatorname{Min}\left\{D_{T}\left(P, X_{1}\right), D_{T}\left(P, X_{2}\right)\right\}$

Uma vez que $X_{1} \in X_{2}$ são dados por:

$X_{1}\left(x_{0},-\frac{a x_{0}}{b}-\frac{c}{b}\right)$

$X_{2}\left(-\frac{b y_{0}}{a}-\frac{c}{a}, y_{0}\right)$

Tem-se que

$D_{T}\left(P, X_{1}\right)=\left|x_{0}-x_{0}\right|-\left|y_{0}+\frac{a x_{0}}{b}+\frac{c}{b}\right|=\left|\frac{a x_{0}+b y_{0}+c}{b}\right|$

$D_{T}\left(P, X_{2}\right)=\left|x_{0}+\frac{b y_{0}}{a}+\frac{c}{a}\right|+\left|y_{0}-y_{0}\right|=\left|\frac{a x_{0}+b y_{0}+c}{a}\right|$

E portanto,

$D_{T}(P, h)=\operatorname{Min}\left\{\frac{\left|a x_{0}+b y_{0}+c\right|}{|b|} ; \frac{\left|a x_{0}+b y_{0}+c\right|}{|a|}\right\}$, se $a \neq 0 \in b \neq 0$

$D_{T}(P, h)=\left|\frac{b y_{0}+c}{b}\right|$ se $a=0$

$D_{T}(P, h)=\left|\frac{a x_{0}+c}{a}\right|$ se $b=0$

Assim, finalmente mostra-se que: 


$$
D_{T}(P, h)=\frac{\left|a x_{0}+b y_{0}+c\right|}{\operatorname{Max}\{|a| ;|b|\}}
$$

Voltando então à parábola, dado um ponto $A\left(x_{a}, y_{a}\right)$ e uma reta $\mathrm{L} \equiv m x+n y+c=0$, tem-se que:

$$
\begin{aligned}
& D_{E}(P, L)=\frac{\left|m x_{a}+n y_{a}+c\right|}{\sqrt{m^{2}+n^{2}}} \\
& D_{T}(P, L)=\frac{\left|m x_{a}+n y_{a}+c\right|}{\operatorname{Max}\{|m|,|n|\}}
\end{aligned}
$$

Assim:

$$
\begin{aligned}
& P_{E} \equiv \sqrt{(x-a)^{2}+(y-b)^{2}}=\frac{\left|m x_{a}+n y_{a}+c\right|}{\sqrt{m^{2}+n^{2}}} \\
& P_{T} \equiv|x-a|+|y-b|=\frac{\left|m x_{a}+n y_{a}+c\right|}{\operatorname{Max}\{|m|,|n|\}}
\end{aligned}
$$

Onde $P_{E}$ \& $P_{T}$ representam de modo algébrico a parábola no modelo euclidiano e no modelo do taxi, respectivamente. A equação $P_{E}$ representada em um sistema de eixos cartesianos nos fornece a seguinte visualização gráfica da parábola:

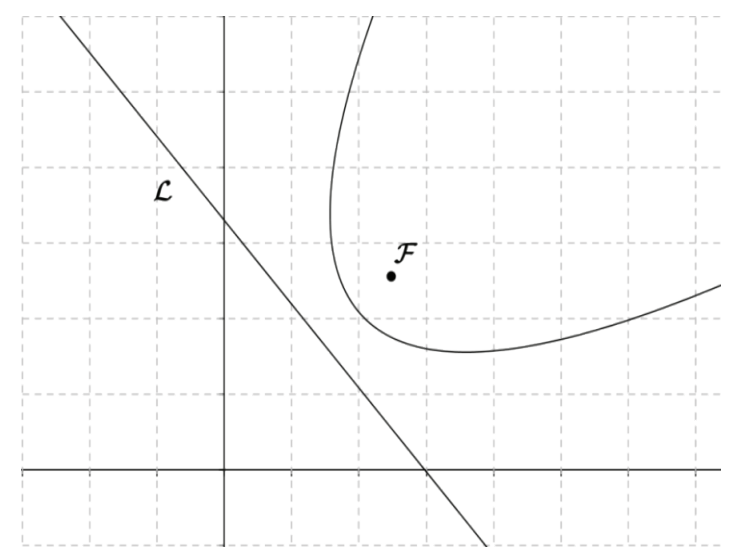

Figura 15 - Parábola Euclidiana

Apresentamos agora um exemplo de como obter a representação gráfica de uma parábola no modelo do taxi.

Exemplo: Dados o ponto $F(0,2)$ e a reta $L \equiv y=-3$, determine os pontos $M(x, y)$ do plano que atendem à equação:

$D_{T}(M, F)=D_{T}(M, L)$

Solução:

Nessas condições, temos então:

$|x-0|+|y-2|=|y+3|$

Analisando a equação acima, temos os seguintes casos:

Para $x<0$ e $y<-3 \rightarrow x=5$ não é uma solução.

$$
\begin{aligned}
& \text { Para } x<0 \text { e }-3 \leq y<2 \rightarrow x+2 y=-1 \text { (a) } \\
& \text { Para } x<0 \text { e } y \geq 2 \rightarrow x=-5(\mathrm{~b}) \\
& \text { Para } x \geq 0 \text { e } y \leq-3 \rightarrow x=-5 \text { não é uma }
\end{aligned}
$$
solução

Para $x \geq 0 e-3 \leq y<2 \rightarrow x-2 y=1$ (c)

Para $x \geq 0$ e $y \geq 2 \rightarrow x=5(\mathrm{~d})$

Desse modo podemos traçar a parábola no modelo do taxi:

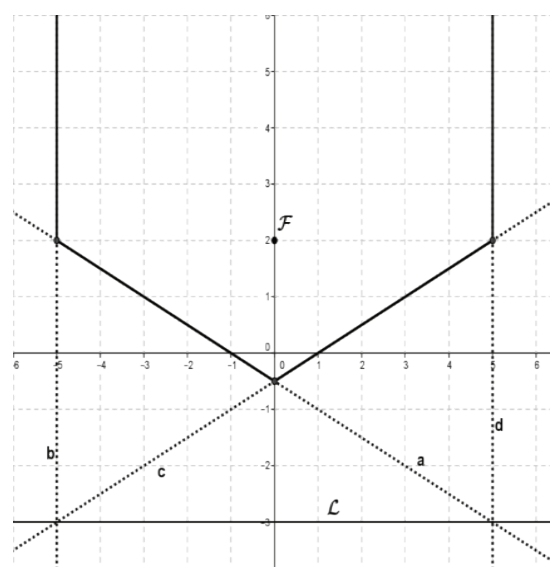

Figura 16 - Parábola do Taxi

\section{Hipérbole}

Dados dois pontos do plano $F_{1} \in F_{2}$, que chamamos de focos, definimos hipérbole como sendo o lugar geométrico dos pontos $M(x, y)$ para os quais o módulo da diferença de suas distâncias a $F_{1} \in F_{2}$ é igual a um valor constante.

Desse modo, dados os pontos $F_{1}(a, b) \in F_{2}(c, d)$ e $k \in R$, temos que:

$$
\begin{aligned}
& H_{E}=\left\{M(x, y) \in R^{2} /\left|D_{E}\left(M, F_{1}\right)-D_{E}\left(M, F_{2}\right)\right|=2 k\right\} \\
& H_{T}=\left\{M(x, y) \in R^{2} /\left|D_{T}\left(M, F_{1}\right)-D_{T}\left(M, F_{2}\right)\right|=2 k\right\}
\end{aligned}
$$

Assim, temos:

$$
\begin{aligned}
& H_{E} \equiv\left|\sqrt{(x-a)^{2}+(y-b)^{2}}-\sqrt{(x-c)^{2}+(y-d)^{2}}\right|=2 k \\
& H_{T} \equiv|| x-a|+| y-b|-(|x-c|+|y-d|)|=2 k
\end{aligned}
$$

Onde $H_{E}$ e $H_{T}$ representam de modo algébrico a hipérbole no modelo euclidiano e no modelo do taxi, respectivamente. A equação $H_{E}$ representada em um sistema de eixos cartesianos nos fornece a seguinte visualização gráfica da hipérbole: 


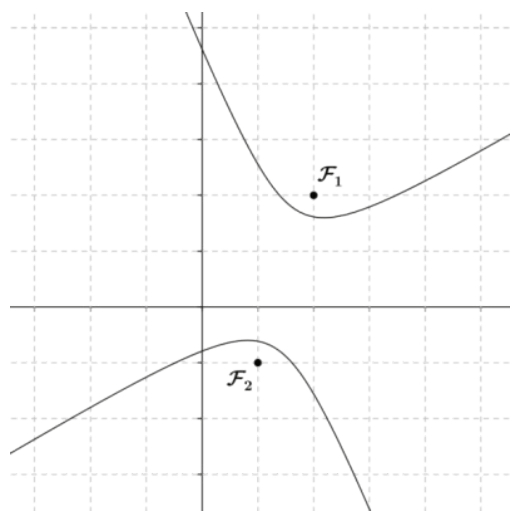

Figura 17 - Hipérbole Euclidiana

Apresentaremos um exemplo de como obter a sua representação gráfica no modelo do taxi.

Exemplo: Dados os pontos $F_{1}(0,0) \in F_{2}(2,-3)$ determine os pontos $M(x, y)$ do plano que atendem a equação:

$$
\left|D_{T}\left(M, F_{1}\right)-D_{T}\left(M, F_{2}\right)\right|=2
$$

Solução:

Nessas condições, temos então:

$$
|| x|+| y|-| x-2|-| y+3||=2
$$

Analisando essa equação, chega-se aos seguintes casos:

$$
\begin{aligned}
& \text { Se } x<0 \text { ey }<-3 \rightarrow \text { não existe solução } \\
& \text { Se } \\
& x<0 \text { e }-3 \leq y<0 \rightarrow y=-7 / 2 \text { ou } y=-3 / 2 \rightarrow y=-3 / 2
\end{aligned}
$$

(a)

Se $x<0$ ey $\geq 0 \rightarrow$ não existe solução

Se

$0 \leq x<2$ e $y \leq-3 \rightarrow x=1 / 2$ ou $x=-3 / 2 \rightarrow x=1 / 2$

(b)

Se $0 \leq x<2 e-3 \leq y<0 \rightarrow x-y=7 / 2$ (c) ou $x-y=3 / 2(d)$

Se

$0 \leq x<2$ e $y \geq 0 \rightarrow x=7 / 2$ ou $x=3 / 2 \rightarrow x=3 / 2$ (e)

Se $x \geq 2$ ey $<-3 \rightarrow$ não existe solução Se

$x \geq 2 e-3 \leq y<0 \rightarrow y=-3 / 2$ ou $y=1 / 2 \rightarrow y=-3 / 2$

(a)

Se $x \geq 2$ ey $\geq 0 \rightarrow$ não existe solução

Tomando agora as soluções das equações acima com suas respectivas condições e traçando em um sistema de eixos cartesianos teremos a representação gráfica dessa hipérbole no modelo do taxi.

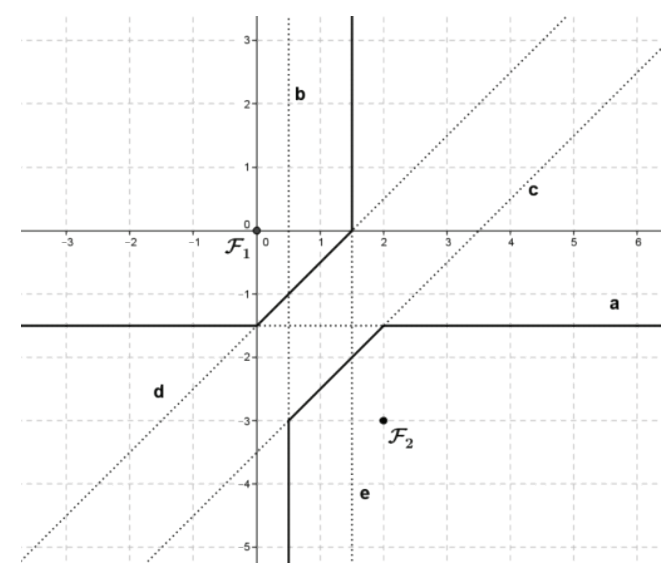

Figura 18 - Hipérbole do Taxi

\section{Considerações Finais}

Mesmo não sendo exaustivo, na apresentação, tanto da Geometria do Taxi quanto no desenvolvimento histórico que possibilitou o aparecimento das Geometrias Não Euclidianas, este trabalho fornece ao professor do Ensino Básico uma forma de observar a matemática sob um aspecto integrador e crítico, possibilitando que o mesmo possa refletir sobre os processos de ensino e as possibilidades que a Geometria fornece como objeto de aprendizagem.

A inegável característica da Geometria em modelar o ambiente permite seu uso como objeto de aprendizagem de compreensão natural e lúdica. A Geometria do Táxi é um modelo natural para a geografia urbana e seu conhecimento possibilita, em consonância com os PCN's, a construção de outras propostas motivadoras, interdisciplinares e próximas do cotidiano do aluno, possibilitando quebra de paradigmas e incentivando abordagens mais críticas e desenvolvimentos mais consistentes da aprendizagem.

Esperamos com esse trabalho, que o professor de matemática do Ensino Básico, abra novos caminhos em sua prática docente e se sinta motivado em criar suas próprias estratégias, pesquisando novos conceitos de aprendizagem.

\section{Referências}

AABOE, A.. Episódios da História da Matemática; tradução: João Bosco Pitombeira. 3 a ed. Rio de Janeiro: SBM, 2013. 191 p. (Coleção Professor de Matemática; 18). 
BICUDO, I. Os Elementos/Euclides; tradução e introdução. São Paulo: Editora UNESP, 2009.

BRASIL, MINISTÉRIO DA EDUCAÇÃO, Parâmetros Curriculares Nacionais para $\mathrm{O}$ Ensino Da Matemática, Brasília, 1998.

KRAUSE, E. F. Taxicab Geometry. New York: Dover Publications Inc, 1986 ( edição original 1937).

MLODINOW, L.. A Janela de Euclides: a história da geometria das linhas paralelas ao hiperespaço. Tradução de Enézio E. de Almeida Filho. São Paulo: Geração Editorial, 2008.

ROQUE, T.. História da Matemática: uma visão crítica, desfazendo mitos e lendas. Rio de Janeiro: Zahar, 2012.

SACCHERI, GEROLAMO. Euclides Vindicatus. Tradução de George Halsted. New York: Dover Publications, 1986

TOMEI, C.. Euclides - A Conquista do Espaço; $2^{2}$ Edição - São Paulo: Odysseus Editora, 2006. (Imortais da Ciência/ Coordenação Marcelo Gleiser). 